\title{
Sensoreinsatz und Datenanalyse in der Landwirtschaft
}

Dr. sc. agr. Johanna Link-Dolezal*, M.Sc. Christoph Zecha, Prof. Dr. Wilhelm Claupein Universität Hohenheim, Institut für Kulturpflanzenwissenschaften, Fg. Allgemeiner Pflanzenbau (340a), Fruwirthstr. 23, 70599 Stuttgart-Hohenheim

Tel.-Nr., E-Mail-Adresse(n): 0711 - 459 22373, Johanna.Link@uni-hohenheim.de

\section{Landwirtschaft und Precision Farming}

Die landwirtschaftliche Produktion hat sich in den letzten Jahren in vielerlei Hinsicht gewandelt. So spielt der Einsatz von Elektronik und Sensoren in der heutigen Landwirtschaft auf den unterschiedlichsten Ebenen eine immer bedeutendere Rolle.

Angefangen bei dem Schlepper zur Bestellung des Feldes, der heutzutage mit einer Vielzahl von Sensoren ausgerüstet ist, die den aktuellen Zustand, die jeweiligen Einstellungen oder den Verbrauch von Betriebsmitteln während des Einsatzes erfassen (Timpe, 2007). Auch bei der Betriebsführung wird eine automatische Datenerfassung angestrebt, um Maschinenzeiten und Ausbringmengen, sowie Erntemengen direkt erfassen und in sog. Schlagkarteien dokumentieren zu können. Hierzu bedarf es eines entsprechend ausgerüsteten Schleppers und entsprechende Anbaugeräte, wie sie mittlerweile auf vielen landwirtschaftlichen Betrieben zu finden sind.

Der Einsatz weiterer Sensoren spielt insbesondere beim sog. Precision Farming eine große Rolle, da hier im Gegensatz zur üblichen Landbewirtschaftung nicht das gesamte Feld als eine Managementeinheit betrachtet wird, sondern die unterschiedlichen Teilflächen innerhalb eines Feldes entsprechend ihrer Wachstumsbedingungen für die Kulturpflanze einzeln betrachtet werden. Demnach werden die notwendigen Managementmaßnahmen nicht auf ein Feld bezogen, sondern auf Teilflächen innerhalb des Feldes. Das vorranige Ziel von Precision Farming ist die Optimierung der Bestandesführung vor dem Hintergrund variierender Wachstumsbedingungen innerhalb des Feldes. Für den Anbau von Kulturpflanzen sind verschiedene Maßnahmen (von Aussaat bis zur Ernte) über das Jahr verteilt auf dem Feld durchzuführen, um den Kulturpflanzenbestand zu dem gewüschten Ertrag mit entsprechender Qualität zu führen.

Durch den Einsatz geeigneter Sensoren kann der Landwirt bei der Bestandesführung (Anonym, 2011) entlastet bzw. bei der Umsetzung von Maßnahmen unterstützt werden. Alle Maßnahmen von der Bodenbearbeitung, über die Aussaat, den Pflanzenschutz und die Düngung an die unterschiedlichen Wachstumsbedingungen im Feld anzupassen, sowie die georeferenzierte Erfassung von Ertrags- und anderen Sensordaten sind zusammen mit dem Einsatz von Spurführungssystemen die zentralen Bereiche des Precision Farming. Die Einsparung von Betriebsmitteln, der Schutz der Umwelt und der sorgsame Umgang mit den natürlich verfügbaren Ressourcen stehen dabei im Vordergrund deren Anwendung. Diese Art der teilflächenspezifischen Bestandesführung im Precision Farming wird maßgeblich durch den Einsatz geeigneter Sensoren unterstützt und optimiert.

\section{Sensoreneinsatz}

Im Rahmen wissenschaftlicher Forschungen werden neue Sensoren für den den Einsatz in Precision Farming entwickelt oder auf dem Markt verfügbare Sensoren hinsichtlich ihrer Eignung zur Optmirung der Bestandesführung getestet. Ein wichtiger Aspekt, um die Sensoren neben der Forschung später in der praktischen Landwirtschat einzusetzen, ist die Möglichkeit die Sensoren online zu betreiben und somit die aus den Messwerten abgeleitete Information direkt an ein angebautes Gerät (z.B. Düngerstreuer oder Pflanzenschutzspritze) weiterzuleiten. Nur unter dieser Voraussetzung ist es möglich in einem Arbeitsgang die notwengigen Daten zu erfassen, diese zu analysieren und gleichzeitig die Gerätesteuerung den variablen Wachstumsbedingungen innerhalb des Feldes anzupassen.

Rhode (2008) beschreibt die Anforderungen an online Sensoren im Agrarberich mit folgenden fünf Kriterien:

- Integrierbarkeit (leichte Integration in vorhanden Abläufe, einfache Instalation, Handhabung, Wartung)

- $\quad$ Robustheit (Anforderungen liegen im Militärbereich, teilweise darüber)

- Logistik der Probenahme (entscheidet über den späteren Einsatzzweck der Applikation. Vereinfachtes handling sicher Effizienz der Lösung) 
- Kalibrationsbasis (bietet breit angelegt Anwendungssicherheit in diversen Einsatzbereichen und unter extremen Umwelteinflüssen)

- Kosteneffizienz (sehr geringe „break even“ Schwelle. Nutzwert steht an erster Stelle).

Zahlreiche kommerzielle Systeme von Herstellern erfüllen diese Kriterien. Viele verfügbaren Systeme verarbeiten die gemessenen Daten und senden die Steuerbefehle direkt an das jeweilige Anbaugerät. Einflussnahme durch den Landwirt bzw. Anwender kann beispielsweise noch über die Kalibrierung sowie die Eingabe manueller Korrekturfaktoren beeinflusst werden.

In der folgenden Tabelle (Tab. 1) werden einige auf dem Markt verfügbare Sensoren zur teilflächenspezifischen Optimierung der Bestandesführung dargestellt:

Tab. 1: Überblick über einige am Markt verfügbare Sensoren zur Umsetzung teilflächenspezifischer Bestandesführung.

\begin{tabular}{|c|c|c|c|c|c|}
\hline \\
\hline Maßnahme & Ziel & Sensor & Funktionsweise & Messwert & Hersteller \\
\hline Düngung & $\begin{array}{l}\text { Anpassung der Appli- } \\
\text { kationsmenge (Stickstoff- } \\
\text { düngung, Halmstabili- } \\
\text { satoren, Fungizide) an } \\
\text { Bestandesdichte und } \\
\text { Chlorophyll }\end{array}$ & $\begin{array}{l}\text { Yara N- } \\
\text { Sensor }\end{array}$ & $\begin{array}{l}\text { Passives Spektro- } \\
\text { meter }\end{array}$ & $\begin{array}{l}\text { Spektralwerte, } \\
\text { Indicees }\end{array}$ & Yara \\
\hline Düngung & $\begin{array}{l}\text { Anpassung der Appli- } \\
\text { kationsmenge an die } \\
\text { Stickstoffversorgung und } \\
\text { das Ertragspotential im } \\
\text { Bestand }\end{array}$ & ISARIA & $\begin{array}{l}\text { Spektrometer unter } \\
\text { Berücksichtigung } \\
\text { von historischen } \\
\text { Ertragskarten }\end{array}$ & $\begin{array}{l}\text { Spektralwerte, } \\
\text { Indicees }\end{array}$ & Fritzmeier \\
\hline Düngung & $\begin{array}{l}\text { Anpassung der Appli- } \\
\text { kationsmenge an die } \\
\text { Stickstoffversorgung }\end{array}$ & $\begin{array}{l}\text { Green- } \\
\text { Seeker }\end{array}$ & $\begin{array}{l}\text { Aktiver } \\
\text { Infrarotsensor }\end{array}$ & Spektralwerte & Trimble \\
\hline $\begin{array}{l}\text { Düngung, } \\
\text { Pflanzenschutz }\end{array}$ & $\begin{array}{l}\text { Anpassung der Appli- } \\
\text { kationsmenge anhand } \\
\text { einer Regelkurve }\end{array}$ & $\begin{array}{l}\text { CROP } \\
\text { Sensor }\end{array}$ & $\begin{array}{l}\text { Aktives Spektro- } \\
\text { meter }\end{array}$ & $\begin{array}{l}\text { Spektralwerte } \\
\text { zur Ermittlung } \\
\text { der Regelkurve }\end{array}$ & Agrocom \\
\hline $\begin{array}{l}\text { Düngung, } \\
\text { Pflanzenschutz }\end{array}$ & $\begin{array}{l}\text { Anpassung der Appli- } \\
\text { kationsmenge (Stickstoff- } \\
\text { düngung, Halmstabili- } \\
\text { satoren, Fungizide) an } \\
\text { Bestandesdichte }\end{array}$ & CROP-Meter & $\begin{array}{l}\text { Mechanisch, } \\
\text { Pendelsensor }\end{array}$ & $\begin{array}{l}\text { Relative } \\
\text { Bestandes- } \\
\text { unterschiede }\end{array}$ & $\begin{array}{l}\text { Agrocom, } \\
\text { Müller } \\
\text { Elektronik }\end{array}$ \\
\hline Pflanzenschutz & $\begin{array}{l}\text { Anpassung der Appli- } \\
\text { kationsmenge (Herbizide) } \\
\text { an Unkrautvorkommen }\end{array}$ & WeedSeeker & Optischer Sensor & $\begin{array}{l}\text { Bild- } \\
\text { auswertung }\end{array}$ & Trimble \\
\hline Ernte & $\begin{array}{l}\text { Erfassen von Inhalts- } \\
\text { stoffen (Feuchte, Trocken- } \\
\text { substanz, Trockenmasse- } \\
\text { erträge) }\end{array}$ & $\begin{array}{l}\text { Harvest Lab, } \\
\text { Corona }\end{array}$ & Nahinfrarosensor & Spektralwerte & $\begin{array}{l}\text { Zeiss, John } \\
\text { Deere }\end{array}$ \\
\hline
\end{tabular}

Darüber hinaus gibt es zahlreiche Forschungsarbeiten, in denen weitere Sensoren zur Ermittlung von unterschiedlichen Wachstumsbedingungen im Feld getestet wurden. Die Arbeiten decken viele Maßnahmen der Bestandesführung (von Aussaat bis Ernte) ab. Die Arbeit von Pforte zeigt beispielsweise die Einsatzmöglichkeit eines Kamera-Sensorsystems zur teilflächenspezifischen Steuerung der Arbeitstiefe bei konservierender Bodenbearbeitung (Pforte, 2010). Ziel der Arbeit war durch die teilflächenspezifische Bewirtschaftung einen optimalen Kompromiss aus Feldaufgang und Erosionsschutz zu finden, indem die Arbeitstiefe des Grubbers von einem Kamera-Sensorsystem gesteuert wurde. In Arbeiten von Knappenberger und Köller (2011) wurde der Einsatz einer Einzelkornsämaschine zur variablen Saatgutablage (Saattiefe) in Mais getestet. Hintergrund war die teilflächenspezifische Optmierung des Feldaufgang bei unterschiedlicher Wasserversorgung im Feld. Die Autoren Maleki et al. (2007) zeigen, wie ein online Sensor im Bereich der sichtbaren und nahinfraroten Spektralmessung zur Anpassung der Phoshatdüngung (Grunddüngung) eingesetzt werden kann. Ein weiterer Aspekt von Forschungsarbeiten ist die Erfassung der Produktqualität innerhalb eines Feldes. Eine Möglichkeit der Nahinfrarot-Spektroskopie zur Erfassung von Protein-, Stärke- und Ölgehalten im Korn. Weitere Einsatzmöglichkeiten für Sensoren sind auf dem Gebiet der Automatisierung und dem Einsatz von Robotern zu sehen.

Grundsätzlich ist zum Thema Sensoreinsatz anzumerken, dass bei dem Einsatz einzelner, sehr spezifischer Sensoren es durchaus die Möglichkeit zur Fehlinterpretation der Daten gibt. Die Ursache 
hierfür liegt in der Tatsache begründet, dass unterschiedliche Wachstumsbedingungen im Feld z.T. ähnliche Sensorsignale auslösen können. Diese Problem gilt es bei der Dateninterpretation zu berücksichtigen.

\section{Datenanalyse}

Die Analyse der im Feld gesammelten Daten erfolgt auf dem landwirtschaftlichen Betrieb meist mit Schlagkarteisoftware, die ebenfalls zum Flächenmanagement benutzt werden kann. Landmaschinenhersteller wie Claas (Anonym, 2012a) oder John Deere (Anonym, 2012b) bieten diese Systeme direkt zum Verkauf als Desktop Lösung an, andere Lösungen, wie beispielsweise von der Firma BASF (Anonym, 2012c) haben ein serverbasiertes Online-Angebot. Sie dienen der Entscheidungsfindung und erleichtern dem Landwirt das Management seiner Applikations- und Ertragsdaten sowie die Planung kommender Einsätze anhand visueller Karten.

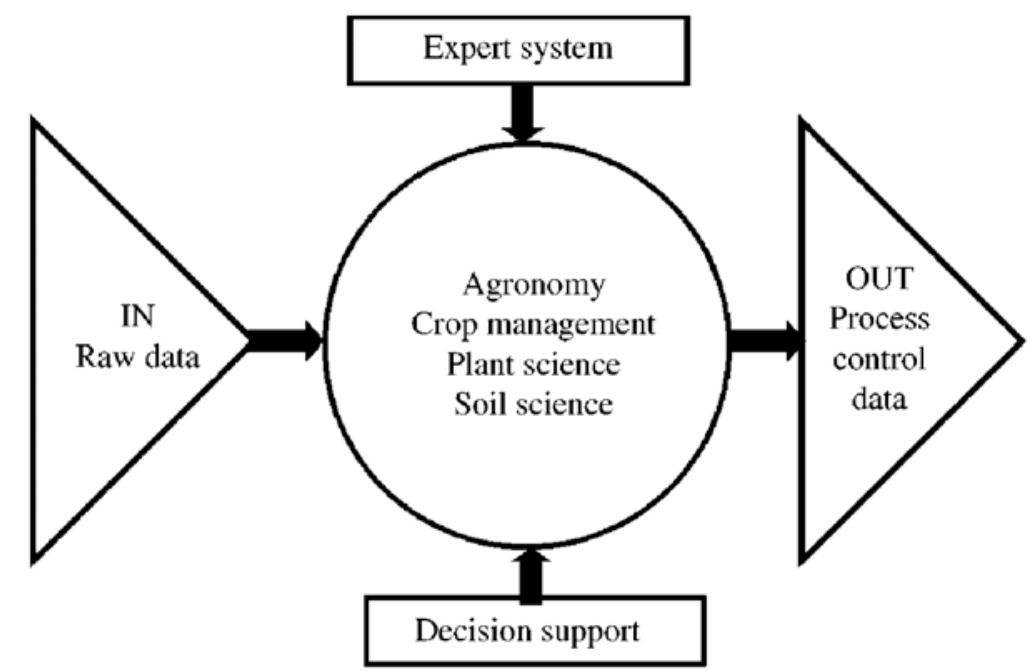

Abb. 1: Informationsfluss und Datenanalyse (Stafford, 2000).

Bisher werden die meisten Entscheidungen auf Basis des Ertrags abgeleitet, als wichtigstem monetärem Kennwert in der landwirtschaftlichen Produktion. Das Potential ist in diesem Bereich noch lange nicht ausgeschöpft. Zur Berücksichtigung anderer Felddaten, wie denen des EM38 zur Bodenleitfähigkeit oder aus Luftaufnahmen fehlt zumeist entweder die technische Ausstattung zur Umsetzung, das Wissen zur Interpretation dieser Daten oder in vielen Fällen einfach nur die Zeit für die Ableitung der Ergebnisse in Entscheidungen. Hier bedarf es neuer Modelle, ganzheitlicher Werkzeuge, die mehr Daten von der gleichen Fläche mit in ihre Berechnungen einbeziehen können und gleichzeitig dem Anwender die Ergebnisse einfach in Anwendungsentscheidungen ausgeben (Abb. 1).

Das laufende Projekt iGreen (Anonym, 2012d) beschäftigt sich genau mit der ähnlichen Thematik mehrere Informationsquellen, in diesem Fall sowohl öffentlicher als auch privater Herkunft, in einem Entscheidungssystem zu vereinen und u.a. dem Anwender diese Daten, z. B. via eines Smartphones leicht zugänglich zu machen.

Von der Statistik geforderte randomisierte Versuchsanlagen mit mehrfacher Wiederholung zur Auswertung von Tests und Versuchen können so in der praktischen Landwirtschaft kaum realisiert werden. Grundlagenforschung auf diesem Gebiet wird deshalb hauptsächlich an Universitäten und Forschungseinrichtungen betrieben, wo dementsprechende Infrastruktur vorhanden ist und wo das gewonnene Wissen leicht verständlich aufbereitet wird, um es in der Praxis zu adaptieren. Die Datenanalyse findet hier auf einer tiefgreifenderen Ebene statt, als das in der praktischen Landwirtschaft anwendbar wäre.

Ob das Feld nach der Datenverarbeitung in mehrere große Managementzonen eingeteilt wird, erkennbar an landschaftlichen Elementen oder Gegebenheiten, oder aber in viele kleine Teilflächen auffindbar durch Satellitenortung, hängt von der technologischen Ausstattung des Betriebes ab. Hier spielt insbesondere die Tatsache, in wie weit der Landwirt GPS Technik verfügbar und mit seinen Maschinen verknüpft hat und mit welcher Genauigkeit er die Anwendungen durchführen kann. Dies beeinflusst auch die Auflösung der späteren Datenanalyse, da Signalgenauigkeiten von unkorrigiertem GPS mit 3-5 m Genauigkeit bis hin zur Real Time Kinematik (RTK) mit -/+ 2,5 cm verfügbar sind (Stafford, 2000). 
Im Projekt SenGIS an der Universität Hohenheim steht für solchen Precision Farming Versuche zum einen die fahrbare Multisensorplattform Sensicle zur Verfügung, sowie zum anderen ein Modellflugzeug als weiterer Sensorträger, ein sogenanntes UAS (unmanned aerial system). Beide Sensorplattformen werden simultan im Feld eingesetzt, so dass die erhobenen Daten direkt miteinander verglichen werden können (Abb. 2). Durch die hohe räumliche Auflösung bedarf es geeigneter Analysemodelle zur Interpretation der Daten und der teilflächenspezifischen Ableitung von Managementempfehlungen (Keller et al., 2011).

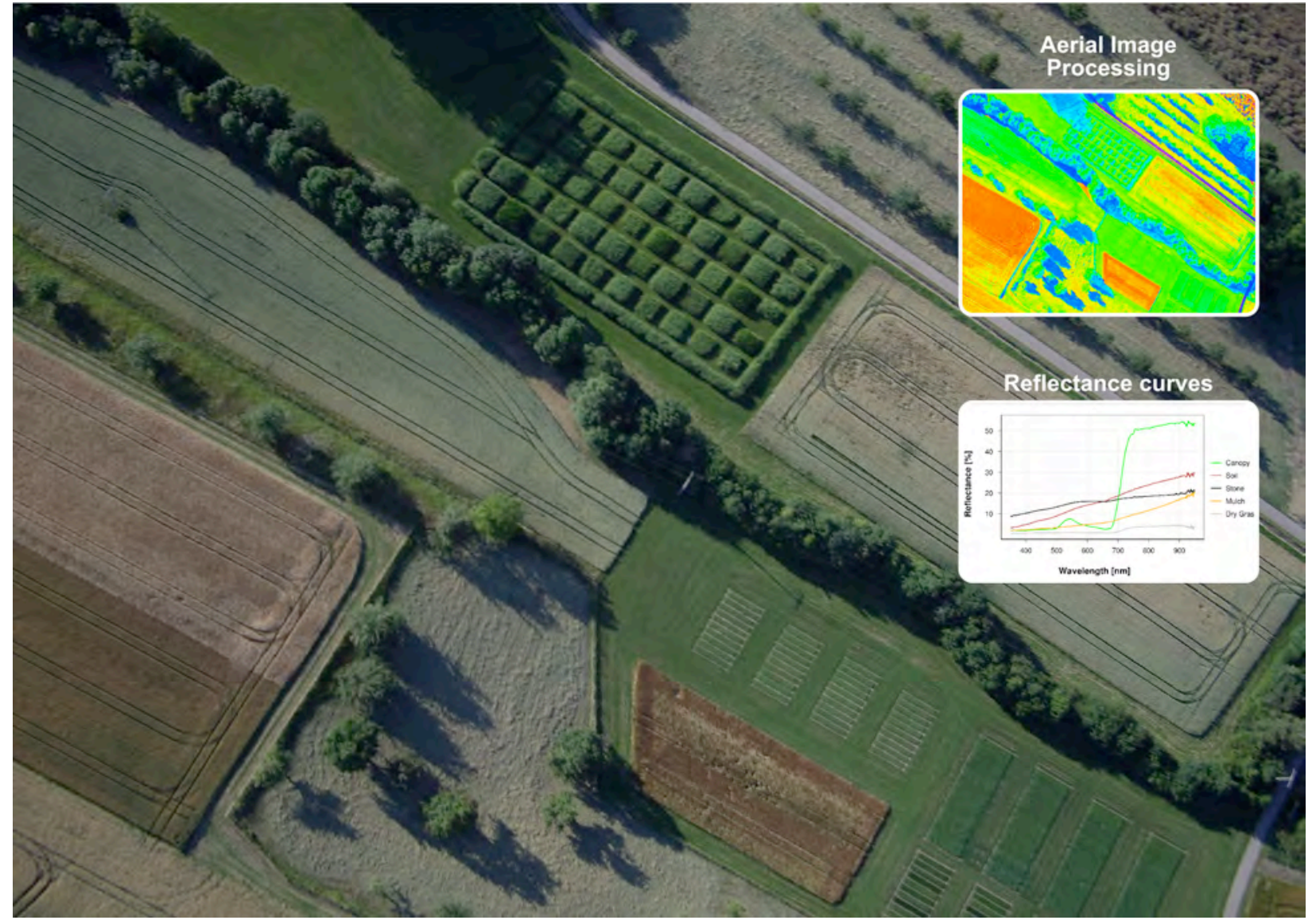

Abb. 2: RGB Bild als Beispiel für weitere Auswerteschritte.

Die Verrechnung und Auswertung der Daten zur Sensorfusion wird mit den Statistikprogrammen R, SAS, SPSS oder SigmaPlot durchgeführt. Data Mining Software wie beispielsweise RapidMiner ist geeignet neue, bisher unbekannte Zusammenhänge zwischen den Versuchsfaktoren und Versuchsdaten aufzuzeigen (Abb. 3). Sog. Sensor Fusion Ansätze spielen dabei eine immer größere Rolle in der Landwirtschaft, d.h. es werden mehrere Sensoren bzw. deren gewonnene Daten miteinander verschmolzen, so wie dies in vielen Bereichen des Flugzeug- und Fahrzeugbau schon seit langem geschieht (Elmenreich, 2002).

Dies kann auf verschiedenen Konfigurationsniveaus stattfinden (Durrant-Whyte, 1988):

- Redundant/Kompetitiv: liefern gleiche Information, z. B. bei Fail-Safe Systemen.

- Komplementär: nicht direkt abhängig voneinander, geben zusammen aber ein besseres Gesamtergebnis, z. B. Spektrometer und Fluorometer.

- Koordiniert: unabhängige Sensoren, im Verbund jedoch unerlässlich, z. B. Stereokamera. 


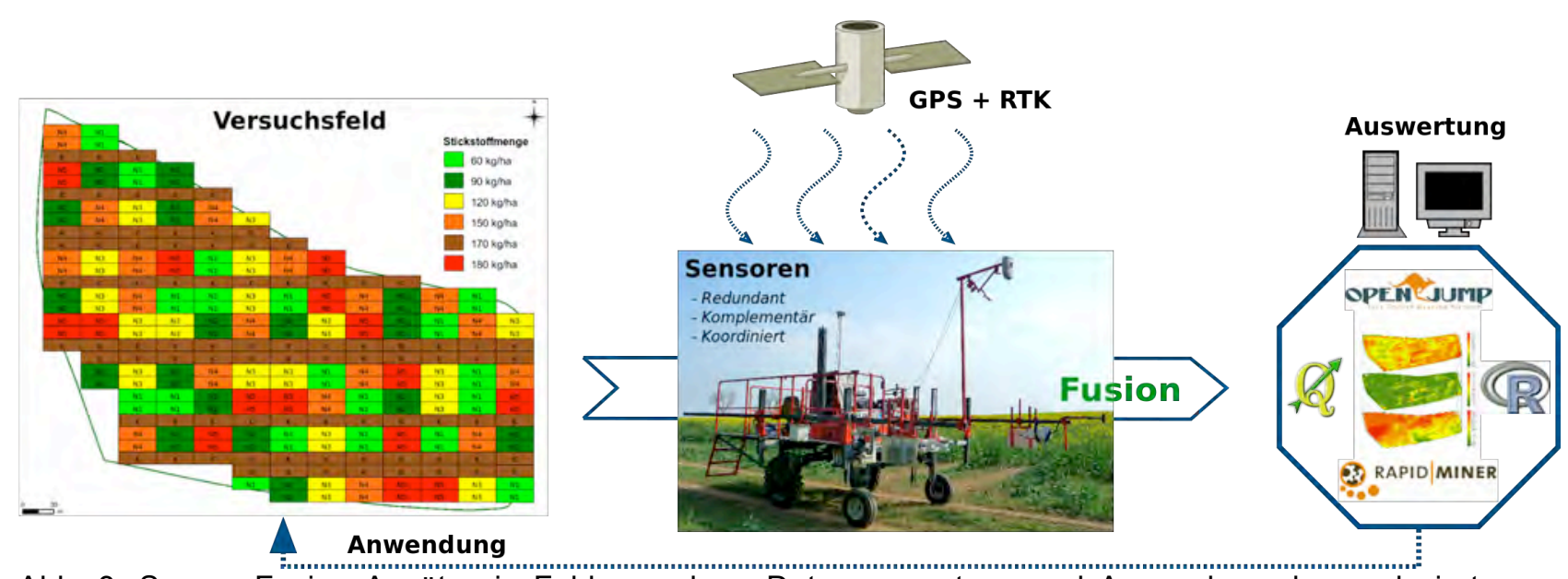

Abb. 3: Sensor Fusion Ansätze in Feldversuchen, Datenauswertung und Anwendung der analysierten Zusammenhänge.

Der Einsatz von mehr Sensortechnik bedeutet jedoch auch höhere Systemkosten, die in der Landwirtschaft nicht bereitwillig investiert werden und sich eher bei größeren Betrieben (größer 100 ha) oder im überbetrieblichen Einsatz lohnen (Adamchuk et al., 2010).

Darüber hinaus verlangen die oben dargestellten Programme für schnelle Entscheidungen auf dem Landwirtschaftsbetrieb profundes Wissen über deren Bedienung und Ergebnisgewinnung. Aus diesem Grund sind diese Methoden bislang für den alltäglichen Gebrauch nur bedingt einsetzbar sind.

\section{Schlussfolgerung}

Zahlreiche interessante Sensoren sind auf dem Markt verfügbar und werden zum Teil sehr erfolgreich eingesetzt. Als problematisch kann unter Umständen angesehen werden, dass jeder Sensor nur auf die Erfassung einzelner Merkmale ausgerichtet sind. Hierbei kann es zu Fehlinterpretationen oder auch Fehlentscheidungen kommen (Zillmann et al., 2006). Ziel muss es daher sein, mehrer Sensoren komplementär einzusetzen, um so beispielweise die Ursache fur Unterschiede im Pflanzenwachstum genau zu ermitllen. Hier spielt das Thema Sensorfusion eine bedeutende Rolle.

Die Umsetzung aktueller Forschungsergebnisse in verfügbare Softwarelösungen werden zwar gut umgesetzt, jedoch kann aufgrund der technischen Ausstattung der Betriebe das generierte Wissen bislang nicht komplett umgesetzt werden. Hier besteht noch Potential für Optimierung sowohl in der Ausstattung als auch in der Produktivität.

Die Datenanalyse der kommenden Generation muss über eine einfach zu bedienende grafische Oberfläche dem Landwirt schnell Informationen liefern, Expertenwissen und/oder servergestützte Wissensdatenbanken mit in ihre Berechnungen einbeziehen und bestenfalls von überall per WebApp (Anonym, 2012e) zugänglich sind.

\section{Literatur:}

Adamchuk, V.I., Viscarra Rossel, R.A., Sudduth, K.A., Schulze Lammers, P. 2011. Sensor Fusion for Precision Agriculture, Sensor Fusion - Foundation and Applications, Ciza Thomas (Ed.), ISBN: 978953-307-446-7, InTech, Available from: http://www.intechopen.com/articles/show/title/sensor-fusionfor-precision-agriculture

Anonym. 2011. Bestandsführung. http://www.agrilexikon.de/index.php?id=501

Anonym. 2012b: Dokumentation und Bürosoftware, John Deere Vertrieb, Bruchsal, http://www.deere.de/wps/dcom/de_DE/products/equipment/agricultural_management_solutions/docu mentation/solutions_and_desktop_software/solutions_and_desktop_software.page?, 20.01.2012.

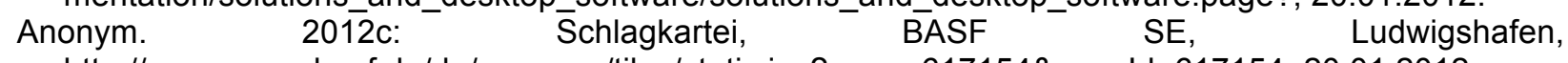
http://www.agrar.basf.de/de/common/tiles/static.jsp?page=617154\&pageld=617154, 20.01.2012.

Anonym. 2012e: Programm ProFlura, ASSW GmbH \& Co. KG, http://www.proflura.de/programm.html, 29.01.2012.

Anonym. 2012a: Komplettlösungen für den Pflanzenbau, CLAAS Agrosystems GmbH \& Co. KG, Gütersloh, http://www.claas-agrosystems.com/de/pflanzenbau/betriebsmanagement-pflanze.html, 20.01.2012.

Anonym. 2012d: Das Projekt, Deutsches Forschungszentrum für Künstliche Intelligenz GmbH, DFKI, Kaiserslautern, http://www.igreen-projekt.de/iGreen/index.php?id=5\&L=0, 21.01.2012. 
Durrant-Whyte, H.F. 1988. Sensor models and multisensor integration, International Journal of Robotics Research, 7, 6, S. 97-113.

EImenreich, W. 2002. An Introduction to Sensor Fusion, Institut für Technische Informatik, Technische Universität Wien, Wien (Österreich).

Griepentrog, H.W. 2011: Eine neue Erfolgsformel? Smart Farming soll die nächste Generation von Precision Farming werden. DLG-Mitteilungen, 126, Frankfurt a.M.; S. 12-16

Keller, M., Zecha, C., Weis, M., Link-Dolezal, J., Gerhards, R., Claupein. W. 2011. Competence center SenGIS - exploring methods for multisensor data acquisition and handling for interdisciplinay research, In: Precision agriculture 2011, S. 491-500, 8th European Conference on Precision Agriculture 2011, Prague, 11-14 July 2011.

Knappenberger, T., Köller, K. 2011. Spatial assessment of the influence of seeding depth on emergence and yield of corn. Journal of Precision Agriculture. DOI: 10.1007/s11119-011-9235-4.

Maleki, M.R., Mouazen, A.M., Ramon, H., Baerdemaker, J. 2007. Optimisation of soil VIS-NIR sensorbased variable rate application system of soil phosphorus. Soil Tillage Res., 94, S. 239-250.

Pforte, F. 2010. Entwicklung eines Online-Messverfahrens zur Bestimmung des Bodenbedeckungsgrades bei der Stoppelbearbeitung zu Mulchsaatverfahren, VDI-MEG 488, ISSN 0931-6264.

Rohde, M. 2008. Sensoren für den agrartechnischen Einsatz. http://www.leibnizinstitut.de/cms/pdf2/rode_sensoren_agrartechnik.pdf

Stafford, J.V. 2000. Implementing Precision Agriculture in the 21st Century, Journal of Agricultural Engineering Research, 76, 3, S. 267-275.

Timpe, R. 2007. Das Geflüster der Sensoren. top agrar, 9/2007, S. 90-96. http://www.topagrar.com/dl/2/1/2/1/4/5/Geflu_776_ster_d_Sensoren.pdf

Zillmann, E., Graeff, S., Link, J., Batchelor, W.D., Claupein, W. 2006. Assessment of Cereal NRequirements derived by Optical on-the-go Sensors on heterogeneous Soils. Agronomy Journal 98, 98; S. 682-690. 